\title{
Image Texture Feature Extraction Method Based on Regional Average Binary Gray Level Difference Co-occurrence Matrix
}

\author{
Jian Yang , Jingfeng Guo
}

College of Information Science and Engineering, Yanshan University, Qinhuang dao, Hebei, China

\begin{abstract}
Texture feature is a measure method about relationship among the pixels in local area, reflecting the changes of image space gray levels. This paper presents a texture feature extraction method based on regional average binary gray level difference co-occurrence matrix, which combined the texture structural analysis method with statistical method. Firstly, we calculate the average binary gray level difference of eight-neighbors of a pixel to get the average binary gray level difference image which expresses the variation pattern of the regional gray levels. Secondly, the regional co-occurrence matrix is constructed by using these average binary gray level differences. Finally, we extract the second-order statistic parameters reflecting the image texture feature from the regional co-occurrence matrix. Theoretical analysis and experimental results show that the image texture feature extraction method has certain accuracy and validity.
\end{abstract}

Index Terms - texture feature; average binary gray level difference; regional co-occurrence matrix; texture feature parameters

\section{INTRODUCTION}

Texture is a visual pattern attribute. It is a property of areas, and consists of sub-patterns which are related to the pixel distribution in a region. So texture is a contextual property and its definition must involve gray values in a spatial neighborhoods.

Texture representation methods can be classified into three categories, namely structural, statistical and multi-resolution filtering methods. Gray Level Co-occurrence Matrix [1] is the most classical second-order statistical method for texture analysis. In practice any texture contains both regular and statistical characteristics. That is why it is difficult to analyze texture by one single method. So in recent years, there exist numerous mixed methods for texture analysis, such as using Gabor filters and co-occurrence probabilities [2]. Wujun [3] used non-sampling wavelet decomposition construct a scale-based concurrent matrix. T.Ojala [4] proposed a kind of effective non-parametric image local texture description methods, Local Binary Patterns (LBP). Then, many variants of LBP have been proposed, such as Local Ternary Patterns [5],

Manuscript Received on June 10, 2011.

E-mail: yangjian095@163.com multi-scale block LBP (MB-LBP) [6], A Bayesian Local Binary Pattern texture descriptor [7].

In this paper, structural method is combined with statistical one. By investigating the traditional gray level co-occurrence matrix which constructs based on pixels, we discover a pair of drawbacks is: (1) it cannot express the change information of the image regional exactly, (2) it is also unable to express the spatial relationship of different local texture patterns in the image. Therefore, a new method is proposed to extract the texture features in this paper, which called regional average binary gray level difference co-occurrence matrix (RABGLD co-occurrence matrix). It can not only capture subtle change of regional gray levels in the image, but also depict texture characteristics of the whole image.

\section{THE CALCULATION OF REGIONAL AVERAGE BINARY GRAY LEVEL DIFFERENCE}

Different regions have different structure features in images. In order to extract the whole image texture characteristics, the texture features of each region should firstly be described. The brightness level at a point in an image is highly dependent on the brightness levels of neighboring points unless the image is simply random noise. Therefore, it is reasonable to describe the regional texture features with the gray level changes of pixels in the neighborhood, and the LBP descriptor is typical. However, LBP only extracts the local texture features from one aspect of the brightness variations, and ignores the overall relationship between the areas. This paper presents a novel method based on the idea of LBP operator, and proposes a new coding rule which is Regional Average Binary Gray Level Difference (RABGLD).

\subsection{Calculating the Regional Binary Gray Level Value}

The joint distribution of all pixels in every $3 \times 3$ neighborhood can be expressed as follows:

$$
G=\left(g_{c}, g_{0}, \cdots g_{7}\right) \text {. }
$$

Where gray value $g_{c}$ corresponds to the gray value of the center pixel of the local neighborhood and $\mathrm{g}_{\mathrm{i}}(\mathrm{i}=0,1, \ldots \ldots, 7)$ corresponds to the gray values of eight neighborhood. Hence, much of the information in the original joint gray level 
distribution (1) about the textural characteristics is conveyed by the joint difference distribution:

$$
T=\left(g_{0}-g_{c}, g_{1}-g_{c}, \cdots g_{7}-g_{c}\right) .
$$

It records the occurrences of two various patterns in the neighborhood of each pixel: one is the neighborhood pixels distribution whose gray values are greater than the center point, and the other one is less. As it concerns the regional textural distribution, that is the changes of gray level, it ignores the case of zero difference. And the joint difference distribution is invariant against gray-scale shifts, that is the texture features are invariant when all of the pixels in the neighborhood plus or minus a value at the same time, so the signs of differences can be also ignored.

Therefore, differences are labeled as follows:

$$
\begin{aligned}
& \left(g_{i}-g_{c}\right)=\left\{\begin{array}{lll}
1 & \text { if } & \left(g_{i}-g_{c}\right)>0 \\
0 & \text { if } & \left(g_{i}-g_{c}\right) \leq 0
\end{array} .\right. \\
& \left(g_{i}-g_{c}\right)=\left\{\begin{array}{lll}
1 & \text { if } & \left(g_{i}-g_{c}\right)<0 \\
0 & \text { if } & \left(g_{i}-g_{c}\right) \geq 0
\end{array}\right.
\end{aligned} .
$$

Now, we get two 8-bit binary sequences. A binomial weight is assigned to each position of neighborhood pixels. We assigned a binomial weight $2^{p}(p=0,1, \cdots, 7)$ or $2^{q}(q=7,6, \cdots, 0)$ to each position whose gray level of neighborhood points is greater or less than the center pixel when counter-clockwise rotating from the right side position of horizontal direction of the center pixel, respectively. Then every value in binary sequence is multiplied by the corresponding weight, and the weighted binary sequences are added.

As a result, we can get Greater Regional Binary Gray Level Pattern (GRBGLP) and Less Regional Binary Gray Level Pattern (LRBGLP), which are related to the neighbor-points whose gray levels are greater or less than the center pixel correspondingly. As shown in Fig. 1.

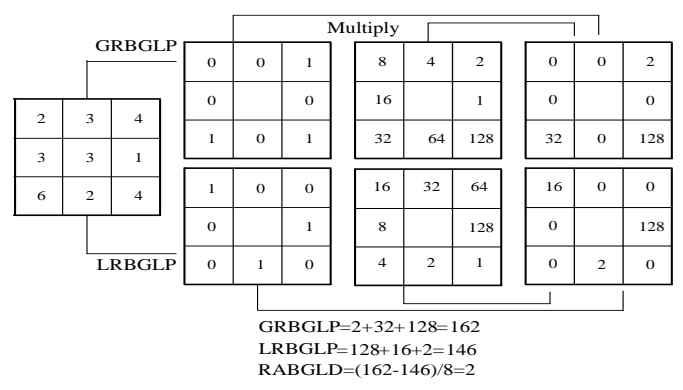

Fig. 1. Calculating the GRBGLP, LRBGLP and RABGLD.

\subsection{Calculating the Regional Average Binary Gray Level Difference}

The RABGLD code for each region of $3 \times 3$ is only produced by integrating the GRBGLP and LRBGLP, and then it can be expressed as (5).

$$
R A B G L D=(G R B G L P-L R B G L P) / 8 .
$$

To construct the completely regional models, we remove the edge pixels of the image, and then compute RABGLD for every $3 \times 3$ region by setting each remaining pixel as the center pixel. It is called "RABGLD Pattern". This pattern represents the distribution of the gray levels or texture characteristics in the region.

For example, the original image and the processed image of original image are shown in Fig. 2. By comparing the RABGLD image with LBP image, it can be found that the extracted labeled image by the method presented in this paper can display the distribution of the image textures clearer and more effective than LBP.

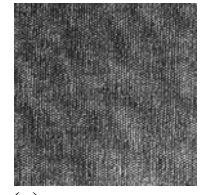

(a)

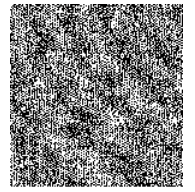

(b)

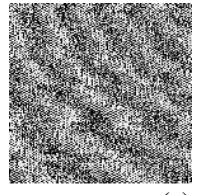

(c)
Fig. 2. (a). The original image,(b):the RABGLD image, (c):the LBP image.

\section{CONSTRUCTING REGIONAL CO-OCCURRENCE MATRIX}

The traditional GLCM reflects the spatial and texture information between the relative positions of different pixels. And this paper aims at constructing the co-occurrence matrix based on distance and direction in the different RABGLD patterns, which is called regional co-occurrence matrix.

Suppose an image which is extracted the RABGLD from the original image is $M \times N$, starting from the region with the RABGLD is $i$ and the coordinate is $(x, y)$, along the distance $(d)$ and orientation $(\theta)$, to the region with the RABGLD is $j$ and the coordinate is $(x+\Delta x, y+\Delta y)$, then we count the simultaneous probability of $(i, j)$, which is $\mathrm{P}(i, j, d, \theta)$. The function can be expressed as (6):

$$
\begin{gathered}
\mathrm{P}(i, j, d, \theta)=\{[x, y],[x+\Delta x, y+\Delta y] \mid f(x, y)=i, f(x+\Delta x, y+\Delta y) \\
=j ; x=0,1, \ldots \mathrm{M} ; y=0,1, \ldots \mathrm{N}\}
\end{gathered}
$$

Where $i, j=(-\mathrm{L}, \mathrm{L}),(\mathrm{L}=255 / 8) ; x$ and $y$ represent the regional coordinates in the image; $\mathrm{M}$ and $\mathrm{N}$ are the size of the image; $\theta$ is the orientation to construct the regional co-occurrence matrix, in generally, the four directions are $0^{\circ}, 45^{\circ}, 90^{\circ}$, and $135^{\circ}$.

\section{TEXTURE FEATURES EXTRACTED FROM REGIONAL CO-OCCURRENCE MATRIX}

Regional co-occurrence matrix contains all texture features information. And analytical method of image texture mentioned in this paper is similar with GLCM. We can extract Energy, Entropy, Contrast, and Correlation second-order statistical characteristics [1] from these regional co-occurrence matrices, and they can identify the textural features efficiently.

(1) Energy (ASM) 


$$
f_{1}=\sum_{i=-L}^{L} \sum_{j=-L}^{L} p^{2}(i, j)
$$

Energy also known as uniformity or ASM, it is a measure of homogeneity of image. In a homogeneous image whose pixels are very similar, there are very few dominant RABGLD transitions, Energy is high. Instead, the value is low.

(2) Entropy (ENT)

$$
f_{2}=-\sum_{i=-L}^{\mathrm{L}} \sum_{j=-L}^{\mathrm{L}} p(i, j) \log _{2} p(i, j) .
$$

This parameter measures the disorder of an image. When the image is not texturally uniform, entropy is very large. Entropy is strongly, but inversely, correlated to energy.

(3) Contrast (CON)

$$
f_{3}=\sum_{n=0}^{L} n^{2}\left[\begin{array}{l}
\sum_{i=-L}^{L} \sum_{j=-L}^{L} p(i, j) \\
|i-j|=n
\end{array}\right] .
$$

Where, $\mathrm{n}$ are the differences between the different RABGLD patterns. If the pairs of regional patterns which have high contrast are more, $\mathrm{n}$ is greater. The contrast feature is a difference moment of the regional co-occurrence matrix and is a measure of the contrast or the amount of local variations present in an image.

(4) Correlation (COR)

$$
f_{4}=\frac{\sum_{i=-L}^{L} \sum_{j=-L}^{L}(i j) p(i, j)-\mu_{1} \mu_{2}}{\sigma_{1}^{2} \sigma_{2}^{2}} .
$$

Where $\mu_{1}, \mu_{2}, \sigma_{1}$ and $\sigma_{2}$ can be defined as follows:

$$
\begin{gathered}
\mu_{1}=\sum_{i=-L}^{L} i \sum_{j=-L}^{L} p(i, j) \\
\mu_{2}=\sum_{j=-L}^{L} j \sum_{i=-L}^{L} p(i, j) \\
\sigma_{1}=\sum_{i=-L}^{L}\left(i-\mu_{1}\right)^{2} \sum_{j=-L}^{L} p(i, j) \\
\sigma_{2}=\sum_{j=-L}^{L}\left(j-\mu_{2}\right)^{2} \sum_{i=-L}^{L} p(i, j)
\end{gathered}
$$

The correlation feature is a measure of regional pattern liner-dependencies in the image. High correlation values imply a liner relationship between the pairs of RABGLD patterns. Thus, the correlation is uncorrelated with energy, entropy and contrast.

\section{EXPERIMENTAL RESULTS AND ANALYSIS}

In order to validate the validity of the method mentioned in this paper, texture features are extracted in the images of Brodatz database [8]. Firstly, we gain an image of RABGLD using the method mentioned in section two. Then we construct the regional co-occurrence matrixes of four directions, i.e. $0^{\circ}$, $45^{\circ}, 90^{\circ}$ and $135^{\circ}$, taking 1 as the spatial distance. Secondly, energy, entropy, contrast, and correlation are computed from the four regional co-occurrence matrixes, as a sixteen-dimensional feature vectors to express image texture features. Finally, we analyze texture feature of image using these features parameters.

Now, the extracted results of texture features in the two group images are given as followed.

\subsection{The first image group}

The first group of three images is the same type and different texture as shown in Fig. 3. And their RABGLD images are extracted from the original images as illustrated by Fig. 4.
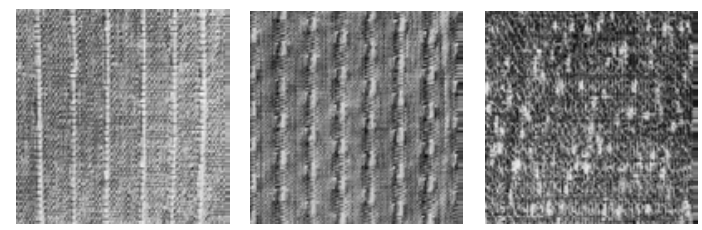

Fig. 3. Wollens raffias.
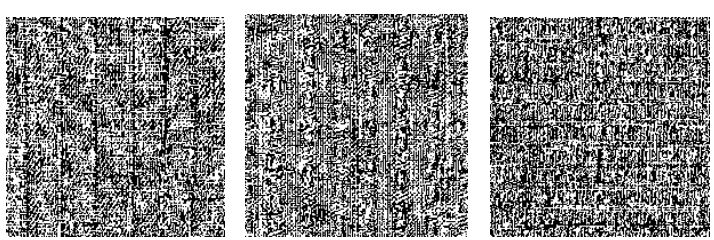

Fig. 4. The RABGLD images of wollens raffias.

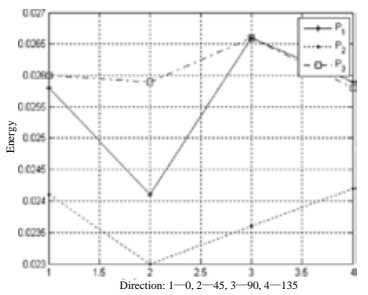

(a) Energy

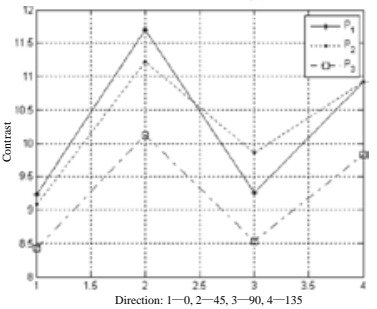

(c) Contrast

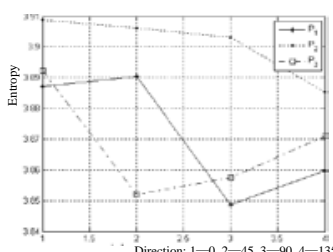

(b) Entropy

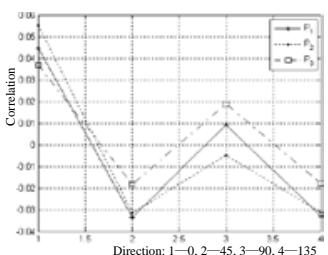

(d) Correlation
Fig. 5. The feature parameters of RABGLD co-occurrence matrix.

The extracted results of texture features are shown in Fig. 5. The curves in (a) show the energy characteristics of the texture. Because the third image has higher energy in all four directions, its RABGLD patterns are similar and texture distribution is more uniform. However, the lowest energy is the second one, so there is a large difference between the regional patterns and it has texture distribution of clutter. The entropy feature is 
shown in Fig. (b), which is the non-uniform and complexity of an image. It is reverse of the energy feature. Contrast is illustrated in (c). And it can be seen from the figure, since there is a large amount of local variations in the $45^{\circ}$ direction compared to other directions, the contrast feature in this direction has consistently higher values. The Fig. (d) shows that the correlation feature is somewhat higher in the horizontal $\left(0^{\circ}\right)$ direction of the three images. And the correlation of third image is higher in $90^{\circ}$ and $135^{\circ}$ than other two images, therefore, the third image has a considerable amount of linear structure along this two directions.

\subsection{The second image group}

The second group images are the different type and texture as shown in Fig. 6.

The second set of experiments is treated with the method which is proposed in this paper and the traditional GLCM, and then texture feature parameters extracted from matrixes are shown in the table I and II. We can learn that the RABGLD co-occurrence matrix based on spatial relationship of regional patterns is approximate to the traditional GLCM method when the whole texture features of image are extracted.

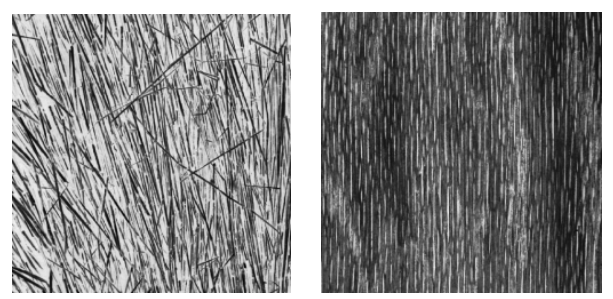

Fig. 6. The Straw and water.

TABLE 1. THE STRAW FEATURE PARAMETERS OF RABGLD CO-OCCURRENCE MATRIX AND GLCM

\begin{tabular}{|c|c|c|c|c|c|c|c|c|c|}
\hline \multirow{2}{*}{ Features } & \multicolumn{2}{|c|}{ RABGLD co-occurrence matrix } & \multicolumn{5}{|c|}{ GLCM } \\
\cline { 2 - 9 } Directions & ASM & ENT & CON & COR & & ASM & ENT & CON & COR \\
\hline $\mathbf{0}^{\circ}$ & 0.0302 & 3.7870 & 9.1388 & -0.0214 & 0.0215 & 4.6156 & 11.6011 & 0.0376 \\
\hline $\mathbf{4 5 ^ { \circ }}$ & 0.0286 & 3.7757 & 9.6539 & -0.0263 & 0.0177 & 4.7466 & 15.9947 & 0.0308 \\
\hline $\mathbf{9 0 ^ { \circ }}$ & 0.0444 & 3.6535 & 6.8476 & 0.0575 & 0.0407 & 4.0127 & 2.6609 & 0.0512 \\
\hline $\mathbf{1 3 5 ^ { \circ }}$ & 0.0347 & 3.7715 & 9.1258 & -0.0193 & 0.0250 & 4.5209 & 9.1357 & 0.0412 \\
\hline
\end{tabular}

TABLE 2. THE WATER FEATURE PARAMETERS OF RABGLD CO-OCCURRENCE MATRIX AND GLCM

\begin{tabular}{|c|c|c|c|c|c|c|c|c|c|}
\hline \multirow{2}{*}{ Features } & \multicolumn{2}{|c|}{ RABGLD co-occurrence matrix } & \multicolumn{4}{|c|}{ GLCM } \\
\cline { 2 - 10 } Directions & ASM & ENT & CON & COR & & ASM & ENT & CON & COR \\
\hline $\mathbf{0}^{\circ}$ & 0.0228 & 3.9027 & 8.9436 & -0.0147 & 0.0281 & 4.1827 & 7.0142 & 0.0688 \\
\hline $\mathbf{4 5 ^ { \circ }}$ & 0.0233 & 3.8762 & 9.5740 & -0.0330 & 0.0275 & 4.1964 & 7.1196 & 0.0681 \\
\hline $\mathbf{9 0 ^ { \circ }}$ & 0.0299 & 3.7427 & 5.3214 & 0.0179 & 0.0620 & 3.2966 & 0.6026 & 0.119 \\
\hline $\mathbf{1 3 5 ^ { \circ }}$ & 0.0228 & 3.8910 & 8.3392 & -0.0353 & 0.0274 & 4.2009 & 7.2348 & 0.0673 \\
\hline
\end{tabular}

\section{CONCLUSION}

This paper presents a new method which extracts second-order statistical parameters to describe texture features from the regional co-occurrence matrix of different RABGLD textural patterns, combining structural method with statistical method. It can extract texture features from single texture or relatively regular textural images, correctly and effectively, which can not only catch the subtle regional gray level changes of image, but also can depict texture pattern characteristics of the whole image. Besides, some disadvantages exist, for example, it cannot extract characteristics from images of texture feature fusion, which is the future work.

\section{ACKNOWLEDGEMENT}

This paper is supported by key basic research program of Hebei province granted No.10963527D.

\section{REFERENCES}

[1] B. Andrea, and P. Flavio, (1995). An Investigation of the Textural Characteristics Associated with Gray Level Co-occurrence Matrix Statistical Parameters. IEEE Transactions on Geoscience and Remote Sensing, vol. 33, pp. 293-304.

[2] D.A. Clausi and D. Huang, (2005). Design-based texture feature fusion using gabor filters and co-occurrence probabilities. IEEE Trans On Image Process, vol. 14, pp. 25-36.

[3] W. Jun and Z. Zhongming, (2001). Scale Co-occurrence Matrix for Texture Analysid Using Wavelet Transform. JOURNAL OF REMOTE SENSING, vol. 5, pp. 100-103.

[4] O. Timo and P. Matti, (2002). Multiresolution Gray-Scale and Rotation Invariant Texture Classification with Local Binary Patterns. IEEE Transactions on Pattern Analysis and Machine Intelligence,vol. 24, pp. 971-987.

[5] T. Xiaoyang and T. Bill (2007). Enhanced local texture feature sets for face recognition under difficult lighting conditions, AMFG.

[6] L. Shengcai and Z. L. Stan, (2007) Learning multi-scale block local binary patterns for face recognition, $I C B$.

[7] H. Chu, A. Timo and P. Matti (2008) A Bayesian Local Binary Pattern Texture Descriptor, ICPR. 19th, pp. 1-4.

[8] The Brodatz texture: image:http://www.ux.uis.no/ .

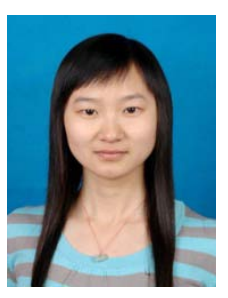

Jian Yang received B.E. degree from the Yanshan University, Qinhuangdao, Hebei,China, in 2009, and now is studying in M.E. Degree in System Structure in the Yanshan University, Qinhuangdao, Hebei,China.

She is currently a M.E. student in the College of Information Science and Engineering, Yanshan University, Qinhuangdao, Hebei,China, since 2009. Her current research interests include image processing and visualization.

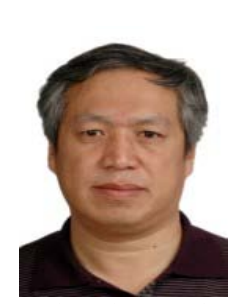

Jingfeng Guo received M.E. Degree in Computer Application from the Northeast College of Heavy Machinery, China, in 1990, and the E.D. degree in Circuits and Systems from Yanshan University, Qinhuangdao, Hebei,China, in 2002.

He has been a Professor in the College of Information Science and Engineering, Yanshan University, Qinhuangdao, Hebei,China, since 2002. He was a Postdoctoral Fellow with the Department of Electrical Engineering, University of Hebei Industry, from 2006 to 2008. He held a visiting researcher position in the Munich Applied Science and Technology University, Germany, in 2005. His research interests include database, data mining, image processing, and visualization. 